\title{
Bats, from Evolution to Conservation
}

\section{José Manuel Mora ${ }^{1}$}

Desde que adquirí mi primer texto sobre murciélagos a mediados de los 80 (Hill y Smith 1984), la literatura sobre este grupo de mamíferos ha crecido exponencialmente, incluidos más libros de texto, científicos y populares. No obstante, me llamó la atención la portada de este libro "Bats from Evolution to Conservation" de John D. Altringham, la cual quizá es poco elaborada, pero con fotos de especies muy familiares para mí: Leptonycteris yerbabuenae, Ectophylla alba y Rhynchonycteris naso, así como un equivalente ecológico muy llamativo como Myzopoda aurita (en el Neotrópico, Thyroptera spp., también tiene discos adhesivos para adherirse a la superficie interna de las hojas nuevas de las heliconias y plantas similares, Mora 2000). Además, el título del libro incluye dos de los tópicos que más me interesan de la biología de los murciélagos, su evolución y su conservación.

Los capítulos sobre evolución y conservación son los que me parecen más importantes. La situación actual de la biodiversidad en general, y de los murciélagos en particular, es de crisis (Primack et al. 2001). Las reservas naturales no tienen el tamaño suficiente para permitir una especiación como la ocurrida hasta ahora y la pérdida de especies es el aspecto más crítico de la crisis de la biodiversidad (Wilson 1992). Los orígenes de esta crisis ambiental radican en el modo de relación establecido por la sociedad industrial con el mundo natural y los seres humanos (Primack et al. 2001). Nuestra visión es homocéntrica y si un organismo no tiene un valor monetario no merece ningún cuidado y si es plaga se le debe eliminar (Altringham 2011).

Aparte de los capítulos antes mencionados, el libro contiene una corta introducción y otros siete capítulos de gran interés sobre la biología de los murciélagos. Así, el libro es un texto para conocedores avanzados de los murciélagos. No obstante, lo considero muy apropiado para estudiantes interesados en la biología de este grupo de mamíferos. El libro es de lectura muy amena, pero con una base científica muy sólida. El autor afirma que el libro tiene como objetivo ayudar a entender a los murciélagos y así entender nuestro impacto sobre ellos (Altringham 2011, p. 266). Sin esta clase de información no podemos practicar una conservación efectiva. Señalaré a continuación de que trata cada capítulo del libro con algunas observaciones específicas en casos particulares.

Capítulo 1. Evolución y diversidad. Este primer capítulo es fascinante, no se quiere dejar de leer. El capítulo contiene varios dibujos que son muy ilustrativos. Los dos factores anteriores lo hacen sentir a uno como a un niño que lee ávidamente su cuento ilustrado favorito.

En la discusión inicial quizá faltó más sobre la clasificación actual y aceptable de los murciélagos, al menos a nivel de suborden. En el capítulo se usa Yimpterochiroptera y Yangochiroptera basado en información reciente de genética molecular. No se discute acerca de otras alternativas tales como Pteropodiformes y Vespertilioniformes. Sin embargo, la discusión sobre la diversidad y relaciones de las familias actuales es muy clara.

La discusión sobre el origen y la evolución de los murciélagos es relativamente corta, pero se hizo con el apoyo de estudios moleculares y de filogenias. La sección de distribución y diversidad de los murciélagos es amplia, ilustrada y amena. El capítulo termina con un corto análisis sobre la alta diversidad y abundancia de los filostómidos.

Capítulo 2. Vuelo. Un capítulo relativamente corto, pero de gran impacto. El autor plantea los principios de la aerodinámica para construir desde ahí sobre el vuelo de los murciélagos. Este camino incluye las ecuaciones y aspectos técnicos similares pertinentes que quizá algunos lectores prefieran obviar. En este sentido, el capítulo comienza un poco pesado para los novatos en física y aerodinámica y al inicio el autor sugiere algunas lecturas en el tema.

El capítulo lleva al lector por los aspectos clave del vuelo tales como su evolución y su funcionalidad. La sección sobre los aspectos ecológicos del vuelo es muy interesante, sobre todo los aspectos correspondientes a la morfología del ala y las

\footnotetext{
${ }^{1}$ Instituto Internacional en Conservación y Manejo de Vida Silvestre (ICOMVIS), Universidad Nacional (UNA), Heredia, Costa Rica. Correo electrónico josemora07@gmail.com
} 
estrategias de forrajeo. En este sentido, los ejemplos e ilustraciones son muy apropiados, aunque el autor no deja de lado su inclinación por los aspectos matemáticos de la aerodinámica.

Capítulo 3. Ecolocación y otros sentidos. Es un capítulo amplio y explicativo, quizá aceptable debido a que la ecolocación es una de las características más distintivas de los murciélagos, aunque es claro que no es exclusiva de este orden ni que todas las especies la poseen (Mora 2000). Contiene unas figuras de aves que, en mi parecer, ocupan mucho espacio para un libro que es de murciélagos. Sin embargo, las explicaciones sobre aspectos como las adaptaciones auditivas y las bases anatómicas de la ecolocación son sencillas y claras.

El capítulo contiene una sección buena con ilustraciones sobre los sonidos de los murciélagos y su detección por medio de aparatos de detección acústica. Esta sección es muy importante debido a que la detección acústica es un tópico que está cobrando relevancia en la actualidad. El autor relaciona estos aspectos con estudios actuales en varios tópicos, incluida la selección de presas. Además, analiza el uso de otros sentidos en las estrategias de la alimentación de los murciélagos.

Capítulo 4. Torpor e hibernación. Es el capítulo más corto del libro (15 páginas). Es un capítulo muy resumido, sobre todo si se compara con el anterior, pero es muy completo. Podría pensarse que el tema no es de amplia utilidad en el trópico. Sin embargo, este tema necesita más énfasis en nuestra región al igual que el tema de la migración (capítulo 7). No sabemos cuánto se usa el torpor en las tierras altas, abundantes en los trópicos. No obstante, hay una corta discusión de ejemplos tropicales con una corta y confusa discusión sobre homotermia y heterotermia en murciélagos, aunque en principio los mamíferos son homotermos (Mora 2000). Esta discusión quizá debió ser más amplia pues parece contradictoria.

Capítulo 5. Reproducción y Desarrollo. Se hace un análisis de los aspectos básicos de la reproducción y el desarrollo, tales como los ciclos reproductivos y la gestación. El apartado sobre la expectativa de vida en los murciélagos quizá pudo ser más amplio debido a la extraordinaria condición de los murciélagos en este sentido en comparación con los mamíferos no voladores.

La sección del comportamiento del apareamiento es relativamente amplia, pero creo que se pudo profundizar más en algunos aspectos. Es cierto, como lo afirma el autor, que se han estudiado muy pocos murciélagos para incluir varios ejemplos en cada patrón de apareamiento. Sin embargo, se pudo describir más a fondo algunos ejemplos que solamente fueron mencionados. El capítulo finaliza con una corta sección sobre la competencia de esperma, el dimorfismo sexual, las señales sexuales y un breve análisis sobre la genética involucrada en estos aspectos. No obstante, el autor puntualiza que retomará el tema en el capítulo 7.

Capítulo 6. Ecología de la alimentación y las perchas. En la biología de los murciélagos se usa el término perchar como traducción de "roosting" para referirse no solo a los sitios de descanso sino a las actividades que ahí se desarrollan. En este sentido, usamos comportamiento o ecología del percheo sin dejar de reconocer la falta del buen español para ello.

La sección sobre la ecología del percheo (incluido el descanso) es amplia, con buenas ilustraciones y muy buenos ejemplos. Se hacen comparaciones sin profundizar en el tema de los equivalentes ecológicos. El autor discute varios aspectos de la ecología del comportamiento del percheo y su relación con la formación de pequeñas y grandes colonias.

La sección de la ecología de la alimentación continúa con la misma calidad de la anterior, pero es interrumpida súbitamente por las láminas a color. ¿Por qué no se pusieron al final del capítulo? Altringham (2011) revisa con ejemplos las estrategias alimentarias de la carnivoría, la piscivoría y la sangrivoría antes de analizar los temas más amplios de la alimentación basada en las plantas y los insectos.

Capítulo 7. Migración, estructura social y estructura poblacional. Aunque se esperaría otra cosa, el capítulo sobre migración comienza con dos ejemplos de filostomátidos. No obstante, no deja de lado los ejemplos más típicos de la migración de los murciélagos, tales como Nyctalus noctula en Europa y Lasiurus cinereus en Norte América. En este devenir, el autor refiere al lector a algunos trabajos de revisión del tema de migración, lo cual es muy valioso, sobre todo para aquellos con intereses específicos.

El autor hace una relación interesante entre la migración y los aspectos tales como las diferencias sexuales y los costos de la migración. Analiza el sistema de navegación usado por los murciélagos así 
como la estructura social y poblacional donde describe aspectos como los refugios y las colonias y la estructura poblacional a pequeña y gran escala. Termina el capítulo con un corto análisis de los efectos históricos de la migración en la estructura poblacional actual de los murciélagos.

La migración no solo tiene consecuencias ecológicas para los murciélagos. En la actualidad, la migración de los murciélagos tiene relevancia en la conservación de las especies. En el Capítulo 9, Altringham (2011, página 265) afirma que para evaluar el efecto de los aerogeneradores en la mortalidad de los murciélagos hay que entender la migración de estos.

Capítulo 8. Biogeografía, macroecología, ecología de la comunidad y las interacciones entre los murciélagos y otros organismos. Son muchos temas para un solo capítulo, pero aunque largo es bueno y entretenido. Quizá el capítulo debió dividirse, por ejemplo, incluir un capítulo solo sobre la biogeografía. Este tema en murciélagos es fascinante y se pudo haber ampliado si se trataba en un capítulo aparte.

Altringham (2011) hace un análisis básico de la relación especies - área y los tópicos relacionados para poner al lector en contexto del amplio resto del capítulo. Lo anterior lo complementa con la situación de las especies en islas, un tópico de gran relevancia en conservación. Además, introduce el tópico de la genética molecular como un método de análisis de los patrones de la colonización que se han dado en el pasado.

En el apartado de la ecología de la comunidad, el autor discute aspectos tales como la partición de nicho y su efecto en las comunidades de murciélagos. Además, aunque de inicio parece fuera de contexto, el apartado de los depredadores y de las presas es de gran importancia en la estructura de las comunidades de los murciélagos. El análisis termina con una muy interesante sección de los murciélagos como presas. También analiza la relación murciélagos - plantas, un tema muy amplio y de gran relevancia para las zonas tropicales y la conservación misma de los bosques lluviosos neotropicales.

Capítulo 9. Conservación. Es la conclusión pues el título del libro es de Evolución a Conservación, pero es a la vez el capítulo más largo del libro (45 páginas). La sección del porqué conservar a los murciélagos parece muy corta e incompleta pues es un tema que tiene mucho por discutir. Esto en cuanto a lo teórico y lo filosófico e incluso en lo práctico. Sin embargo, estos aspectos los va cubriendo el autor con ejemplos claros en las demás secciones del capítulo.

Al inicio del capítulo 9, el autor recomienda algunos libros generales sobre la biología de la conservación lo cual es valioso sobre todo para los lectores con poca experiencia en el tema. Altringham (2011) menciona la extinción de 12 especies de murciélagos que incluye a pteropódidos y a pequeños insectívoros. El autor enlista 11 de estas especies de murciélagos que eran habitantes de islas. La probabilidad de extinción en islas es más común que en las áreas continentales (Primack et al. 2001) y los murciélagos no son la excepción. Ocho de las 12 especies no se han visto en más de 50 años y cuatro más se han extinguido en tiempos más recientes. Desafortunadamente, la lista sigue creciendo y recientemente (2009) se reportó la extinción de dos zorros voladores de Samoa.

El capítulo incluye varios ejemplos de especies de murciélagos del Neotrópico en varios de los subtemas. El autor incluye los ejemplos y menciona que "le dejo a usted a estudiar algunos de los ejemplos en detalle mediante la lectura de los artículos originales". Una de las referencias es la de Schipper et al. 2008. Esta publicación realizada en la prestigiosa revista Science se basa en varias actividades, incluidos algunos talleres de diagnóstico, uno de los cuales fue realizado por el Centro Zamorano de Biodiversidad en el campus de la Escuela Agrícola Panamaericana, Zamorano, Honduras (Evaluación Mundial de Mamíferos [Global Mammal Assessment] para Centroamérica y el Caribe, Zamorano 2008). En el apartado de los murciélagos y los paisajes agrícolas, menciona que algunas plantaciones de café bajo sombra mimetizan al bosque natural y señala como ejemplo de este tipo de ecosistema a las plantaciones aledañas al parque nacional Cusuco en Honduras del cual incluye una fotografía (Figura 9.17a, p. 275).

El autor hace una reflexión sobre la mortalidad de los murciégalos debido a los aerogeneradores. Muy apropiadamente, sugiere que se deben construir turbinas más eficientes, lo cual está siendo investigado en la actualidad, pero hacerlas a la vez menos eficientes para matar animales silvestres. En segundo lugar, señala la necesidad de que seamos menos despilfarradores en nuestro uso de energía, jsi 
usamos la energía más eficientemente, necesitaremos generar menos!

En el capítulo 9 se hace un análisis general de las causas básicas como la deforestación y la fragmentación con ejemplos de murciélagos. El autor provee datos concisos, pero alarmantes, sobre otros problemas como la cacería de murciélagos para comer donde miles de individuos han sido sacrificados, miles de ellos pertenecientes a especies isleñas en peligro de extinción. Además, hay una discusión importante sobre el uso de los murciélagos de las cuevas y las minas, un tema que debe ser relevante en Honduras y otras áreas ya que se ha hecho muy poco o nada para su protección. Por ejemplo, en Taulabé y Agalta el buen manejo de visitantes debe ser prioritario, algo que se hace en las cuevas de Taulabé, pero no tanto en Agalta.

El capítulo contiene una sección de Cambio Climático, muy apropiada, resumida y con buenos ejemplos, incluido el caso de los murciélagos de Monteverde en Costa Rica (Laval 2004). Luego discute otros problemas actuales como la infraestructura basado en dos casos: los parques eólicos y los caminos. La discusión de los parques eólicos se hace con una perspectiva amplia basada en su utilidad y los problemas sobre la biodiversidad.

El capítulo 9 finaliza con una pequeña sección sobre los murciélagos como bioindicadores y señala que al promocionar a los murciélagos como bioindicadores se promueve su conservación. Para este apartado, sugiere la lectura de Jones et al. (2009). Este es un excelente trabajo que nos ha servido de base para escribir un protocolo de muestreo de murciélagos para Honduras. El capítulo finaliza con una lista de cinco organizaciones relacionadas con la conservación de los murciélagos. Sin embargo, existen muchas más organizaciones a este nivel, incluidas varias en Meso y Sur América.

Sobre las posibilidades de extinción en el capítulo 9, el autor recomienda echar mano al principio precautorio, lo cual es muy sensato con un grupo del que de muchas especies se tiene muy poca información. No conocemos los tamaños poblacionales ni sus tendencias de la mayoría de especies de murciélagos. Por ejemplo, prácticamente no tenemos los datos mínimos en los temas mencionados de los murciélagos de Honduras y posiblemente la situación es similar en varios países de Centro América.
Correspondientemente, el libro finaliza con una sección de Referencias (p. 285-317). Ésta es muy amplia, con una gran revisión de la literatura reciente, sobre todo en diversos tópicos de la conservación de los murciélagos. No se debe pretender que el autor fuese escolástico, pero varias publicaciones relevantes del Neotrópico fueron ignorados. Hay que considerar, sin embargo, que quizá el autor no tuvo acceso a estos trabajos.

Cuando terminé de leer el libro ya se le había despegado parte de la tapa y toda la primera sección ya estaba suelta ( $i 12$ páginas!). Un mal trabajo para una editorial de tanto prestigio como Oxford University Press (OUP). No sé si la impresión de OUP de los Estados Unidos o la versión de tapa dura sean de mejor calidad, pero esto es un fallo grave en un libro de más de $\$ 50$.

Las figuras del libro son muy bien hechas, quizá algunas con falta de arte, pero en ellas se ve claramente lo que se quiere enseñar. En la figura 1.15 hay un esquema invertido ya que se señala a Sturnira sp. que claramente es erróneo ya que las especies de este género no tiene uropatagio, mientras que Craseonycteris thonglongyi si tiene uropatagio. Tiene varias figuras que debieron ser a color: 9.14, 9.15, 9.16, incluso $9.17,9.18,9.19$ y 9.20 . Hay ocho láminas con 34 fotos en páginas no numeradas. Están a la mitad del libro (p. 160) pero pudieron incluirse en la página 174, al final del capítulo 6 y así no partir el capítulo. Son buenas fotos, por supuesto, son de Merlin Tutle de Bat Conservation International.

El trabajo editorial en general es bueno con algunas pequeñas fallas. En la página 22 hay todo un párrafo en letra cursiva que claramente no corresponde usar este tipo de letra. La cita de Beckerman et al. 2007 está mala ya que debe ser Anim. Conserv 10: 320-325 en vez de Anim. Conserv 320:320-325. La figura 5.7, aparte de la letra tan pequeña, tiene un error pues se debió escribir "polygyny" en vez de "polygny". La rotulación de la figura 9.20 está bajo las fotos a y b y en la siguiente página están las fotos $\mathrm{b}$ y $\mathrm{c}$, sin rotulación.

Aunque el precio del libro es relativamente accesible, no me gustó su calidad de imprenta. Ahora debo pensar en pagar un reempaste. Sin embargo, a pesar de esto y algunas pequeñas fallas del contenido del libro, indudablemente lo recomiendo. El libro no solo contiene muy buena información sino que esta es actualizada y con gran cantidad de referencias 
bibliográficas en cada sección. En este sentido, el libro es muy apropiado para los amantes de los murciélagos, aficionados 0 profesionales, pero también es muy útil para todos aquellos interesados en la biodiversidad y su conservación.

Me gustó mucho el énfasis y el enfoque de la conservación de los murciélagos. En el capítulo 9, el autor enfatiza que la educación puede ser la herramienta más efectiva para la conservación. Altringham (2011) puntualizó que no hacemos una conservación efectiva entre otras cosas por la pobre comunicación existente entre los investigadores y los practicantes. Este libro pretende ayudar a entender a los murciélagos para apoyar en hacer una conservación más efectiva.

Los dos últimos aspectos son fundamentales para la conservación en general. Además, no solo es necesaria la comunicación entre profesionales, si no enfatizar en la conservación per se. En este sentido, es indispensable la colaboración y la perspectiva clara de que lo importante son los murciélagos y no mi hoja de vida y menos mi ego. La conservación es siempre un acuerdo mutuo, un compromiso entre las partes (Altringham 2011). Los primeros que deben luchar por estos acuerdos son los investigadores y los conservacionistas. Los murciélagos no son propiedad de ningún autor ni de ningún investigador. Al mismo tiempo, el conocimiento generado es de la sociedad en general y como tal debe usarse con el fin de lograr beneficios para ésta. La supervivencia de los murciélagos es a largo plazo un beneficio para la sociedad y a la vez es un compromiso de ésta. "No heredamos esta tierra de nuestros antepasados, la tomamos prestada de nuestros hijos" (Proverbio Sioux).

\section{Literatura Citada}

Altringham, J.D. 2011. Bats, from Evolution to Conservation. Segunda edición. Oxford University Press, Oxford. $324 \mathrm{p}$.

Hill, J.E. y J.D. Smith. 1984. Bats: A Natural History. University of Texas Press, Austin, Texas. $243 \mathrm{p}$.

Jones, G., D.S. Jacobs, T.H. Kunz, M.R. Willig y P.A. Racey. 2009. Carpe noctem: the importance of bats as bioindicators. Endangered Species Research 8: 93-115.
Laval, R.K. 2004. Impact of global warming and locally changing climate on tropical cloud forest bats. Journal of Mammalogy 85:237-244.

Mora, J.M. 2000. Los mamíferos silvestres de Costa Rica. Editorial UNED, San José, Costa Rica. 240 p.

Primack, R., R. Rozzi, P. Feinsinger, R. Dirzo y F. Massardo. 2001. Fundamentos de conservación biológica, perspectivas latinoamericanas. Fondo de Cultura Económica, México D.F. 497 p.

Schipper, J., J.S. Chanson, F. Chiozza, N.A. Cox, M. Hoffmann, V. Katariya, J. Lamoreux, A.S.L. Rodrigues, S.N. Stuart, H.J. Temple, J. Baillie, L. Boitani, T.E. Lacher Jr., R.A. Mittermeier, A.T. Smith, D. Absolon, J.M. Aguiar, G. Amori, N. Bakkour, R. Baldi, R.J. Berridge, J.Bielby, P.A. Black, J.J. Blanc, T.M. Brooks, J.A. Burton, T.M. Butynski, G. Catullo, R. Chapman, Z. Cokeliss, B. Collen, J. Conroy, J.G. Cooke, G.A. B. da Fonseca, A.E. Derocher, H.T. Dublin, J.W. Duckworth, L. Emmons, R.H. Emslie, M. Festa-Bianchet, M. Foster, S. Foster, D.L. Garshelis, C. Gates, M. Gimenez-Dixon, S. González, J.F. González-Maya, T.C. Good, G. Hammerson, P.S. Hammond, D. Happold, M. Happold, J. Hare, R.B. Harris, C.E. Hawkins, M. Haywood, L.R. Heaney, S. Hedges, K.M. Helgen, C. Hilton-Taylor, S.A. Hussain, N. Ishii, T.A. Jefferson, R.K.B. Jenkins, C.H. Johnston, M. Keith, J. Kingdon, D.H. Knox, K.M. Kovacs, P. Langhammer, K. Leus, R. Lewison, G. Lichtenstein, L.F. Lowry, Z. Macavoy, G.M. Mace, D.P. Mallon, M. Masi, M.W. McKnight, R.A. Medellín, P. Medici, G. Mills, P.D. Moehlman, S. Molur, A. Mora, K. Nowell, J.F. Oates, W. Olech, W.R.L. Oliver, M. Oprea, B.D. Patterson, W.F. Perrin, B.A. Polidoro, C. Pollock, A. Powel, Y. Protas, P. Racey, J. Ragle, P. Ramani, G. Rathbun, R.R. Reeves, S.B. Reilly, J.E. Reynolds III, C. Rondinini, R.G. Rosell-Ambal, M. Rulli, A.B. Rylands, S. Savini, C.J. Schank, W. Sechrest, C. Self-Sullivan, A. Shoemaker, C. Sillero-Zubiri, N. De Silva, D.E. Smith, C. Srinivasulu, P.J. Stephenson, N. van Strien, B.K. Talukdar, B.L. Taylor, R. Timmins, D.G. Tirira, M.F. Tognelli, K. Tsytsulina, L.M. Veiga, J-C. Vié, E.A. Williamson, S.A. Wyatt, Y. Xie y B.E. Young. 2008. The status of the world's land and marine mammals: Diversity, threat, and knowledge. Science 322: 225-230.

Wilson, E.0. 1992. The Diversity of Life. Belknap Press of Harvard University Press, Cambridge, Massachusetts. $440 \mathrm{p}$.

Zamorano. 2008. Informe Anual 2008. Annual Report. Valle del Yeguare, Honduras. 46 p.

Recibido para publicación el 19 de mayo de 2013. Aceptado para publicación el 21 de octubre de 2013. 